\title{
Fokale Lungenparenchymveränderungen in der c. Görg konsolidierten Lunge: Ein sonographischer Bildessay
}

\author{
Focal Lesions in the Opacified Lung: A Sonographic Pictorial Essay
}

\section{Zusammenfassung}

Die transkutane Thoraxsonographie hat sich mittlerweile als ergänzende Methode bei röntgenologisch flächenhaften Lungenverschattungen etabliert. Innerhalb von Lungenkonsolidierungen unterschiedlicher Ätiologie lassen sich synchron und metachron fokale Herdbildungen sonographisch darstellen, deren pathomorphologisches Korrelat häufig zunächst unklar ist. Ziel des vorliegenden Bildessays ist es, das sonographische Spektrum von fokalen Herdbildungen und ihre Differenzialdiagnose in der konsolidierten Lunge darzustellen.

\section{Schliuisselwörter}

Ultraschall · Thoraxsonographie · Lungenkonsolidierung

\section{Abstract}

Transcutaneous ultrasound of pleural lesions has been established as a method complementary to conventional X-ray examination. The opacified lung allows the sonographical investigation of the lung parenchyma for synchronous and metachronous focal lesions. The aim of the pictorial essay is to describe the sono graphic patterns and to discuss the differential diagnosis, as well as the pathological correlation of focal lesions in the opacified lung.

Key words

Ultrasound · chest ultrasound · opacified lung
Im Bereich des Thorax hat sich die transkutane Exploration von Thoraxwandprozessen [1], von intrapleuralen Flüssigkeitsansammlungen [2] sowie von peripheren pleurawandständigen Lungenprozessen bewährt [3]. Aufgrund nahezu 100\%iger Schallreflexion an Luft sind der sonographischen Darstellung von Lungenparenchymveränderungen enge Grenzen gesetzt.

Wenn flächenhafte luftverdrängende Lungenprozesse die Lungenoberfläche erreichen, können sie sonographisch dargestellt werden. So ist das sonographische Erscheinungsbild von Lobärpneumonien [4], Atelektasen [5], Lungeninfarkten [6] und Bronchialkarzinomen [7] beschrieben. Eine definitive Beurteilung pathologischer Parenchymveränderungen ist allerdings in den al- lermeisten Fällen nur in Verbindung mit der Röntgenuntersuchung des Thorax und einer Computertomographie möglich. Die flächenhafte fehlende Belüftung von Lungenparenchym führt zunächst zu einer variablen echoarmen Transformation von Lungengewebe im Sinne einer „Hepatisation“ und ermöglicht so die sonographische Detektion von synchron oder metachron entstandenen fokalen Lungenparenchymveränderungen, deren Ätiologie und Dignität häufig unklar ist. Dabei weist das Lungenparenchym einige pathophysiologische Besonderheiten auf, deren Kenntnis für die Beurteilung fokaler Parenchymveränderungen hilfreich ist. Die für die sonographische Darstellung der Lunge notwendige Luftverdrängung ist immer bereits der erste pathologische Prozess, welcher entweder durch verdrän- 


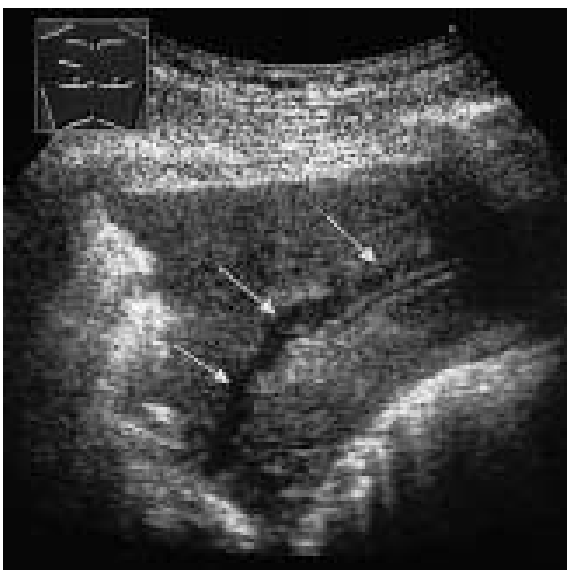

Abb. 1 65-jähriger Patient mit seit 4 Wochen bestehender, bronchoskopisch bestätigter Oberlappenatelektase bei Bronchuskarzinoid. Darstellung einer länglichen, nahezu echofreien gefäßbegleitenden Struktur (Pfeile) dem obstruierten Bronchus entsprechend (Fluidbronchogramm).

Fig. 165 year old patient with central bronchial carcinoid and obstructive atelectasis. On B-mode sonography a echofree longitudinal lesions nearby the vessels (arrow) was seen caused by an obstructive dilated bronchus ("fluid bronchogram").

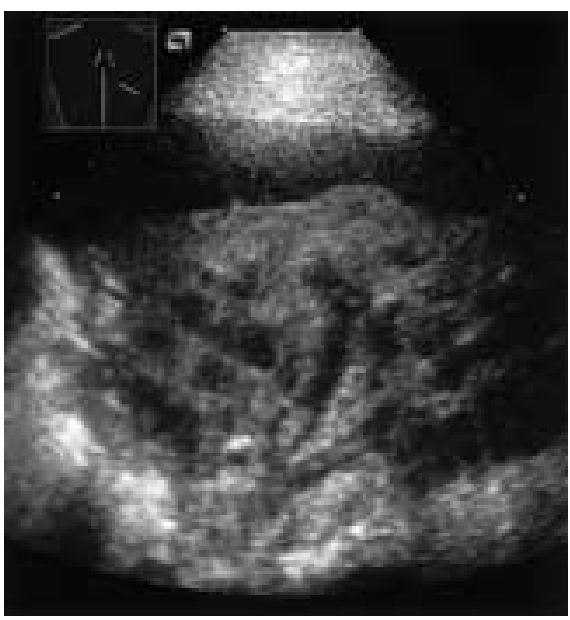

Abb. 2 60-jähriger Patient mit bronchoskopisch bestätigter Unterlappenatelektase bei metastasiertem Nierenzellkarzinom. Darstellung von länglichen, aber auch angeschnittenen rundlichen, nahezu echofreien Bronchiallumina bei chronischer Obstruktion (Fluidbronchogramm).

Fig. 260 year old patient with metastasized hypernephroma and atelectasis of the lower lobe which was confirmed by bronchoscopy. On B-mode sonography longitudinal as well as round echofree lesions were seen caused by a obstructive dilated bronchiogenic tree ("fluid bronchogram").

gende Infiltration (entzündlich/tumorös) oder durch Resorption bei zentraler Obstruktion bedingt ist. Aufgrund des Euler-Lilljestrand-Reflexes kommt es bei fehlender Ventilation zu einer Vasokonstriktion mit konsekutiver Minderperfusion. Auf dieser Grundlage können sich im konsolidierten Lungenparenchym weitere pathologische Prozesse entwickeln, bevorzugt fokale Herdbildungen 1. durch obstruktive bronchiale Retention und 2. durch nutritiv destruierende Liquidefizierung. Dabei erfordert das physiologische Vorkommen von bronchialer Luft eine ortsspezifische Interpretation der sonographischen Befunde. Ähnlich

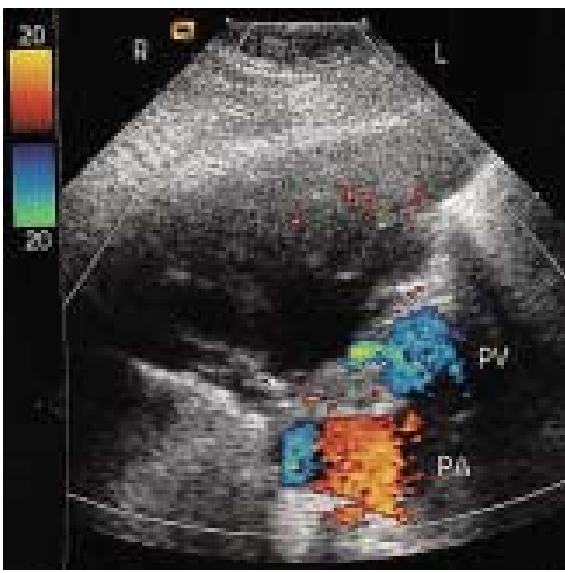

Abb. 3 63-jährige Patientin mit seit 1 Jahr bestehender Oberlappenatelektase bei Z.n. Candidapneumonie. Darstellung eines echofreien Hohlraums, welcher einem pseudozystisch dilatierten Bronchiallumen entspricht ( $\mathrm{PV}=$ Pulmonalvene, $\mathrm{PA}=$ Pulmonalarterie).

Fig. 363 year old patient with a chronic atelectasis of the upper lobe since one year, caused by candida pneumonia. On color Doppler sonography an echofree cyst like lesion was seen caused by a dilated bronchus ( $\mathrm{PV}=$ pulmonary vein, $\mathrm{PA}=$ pulmonary artery).

wie in anderen parenchymatösen Organen werden fokale Herde im Vergleich zum umgebenden Gewebe in prädominant echofrei, echoarm und echoreich eingeteilt.

\section{Prädominant echofreie intraparenchymatöse Herde}

Echofreien Lungenparenchymveränderungen liegt entweder eine zystisch imponierende Erweiterung vorbestehender Hohlraumsysteme (z.B. Bronchien, Pulmonalarterie, Pulmonalvene) oder eine sekundäre Destruktion von Lungengewebe mit Liquidefizierungen zugrunde (z.B. einschmelzender Tumor, postentzündliche Abszessbildung).

Bei länger bestehender zentraler Bronchusobstruktion, sei es durch Tumor, Schleimverhalt oder externe Bronchuskompression durch Lymphome mit konsekutiver Ausbildung einer Atelektase, kann es zu einem Sekretverhalt mit Darstellung von rundlichen, aber auch länglich angeordneten echofreien Bronchiallumina unterschiedlicher Größe kommen, welche auch als „Fluidbronchogramm" bezeichnet werden (Abb.1 und 2). Sie können bei chronischer Obstruktion zu einer pseudozystenartigen Bronchialtransformation im atelektatischen Lungengewebe führen (Abb. 3). Bei zystischen Raumforderungen im Lungenhilus kann mittels Farbdopplersonographie die erweiterte Pulmonalarterie differenzialdiagnostisch abgegrenzt werden (Abb.4). Ein anderes Bild präsentieren polyzystisch einschmelzende Tumoren (Abb. 5). Die Inzidenz von Kavitationen in Lungenkarzinomen wird mit 10\% angegeben [8]. Sie treten bei größeren Tumoren auf. Die Höhlenbildung kann zentral, aber auch peripher auftreten. Grundsätzlich ist auch bei echofreien Formationen eine abszedierende Einschmelzung zu bedenken. Hierbei lassen sich Mikroabszesse von Makroabszessen differenzieren. Einen Sonderfall stellt das Lungengangrän dar mit postpneumatischer Entwicklung von nekrotischen Lungenfragmenten innerhalb eines Lungenabszesses (Abb. 6). 


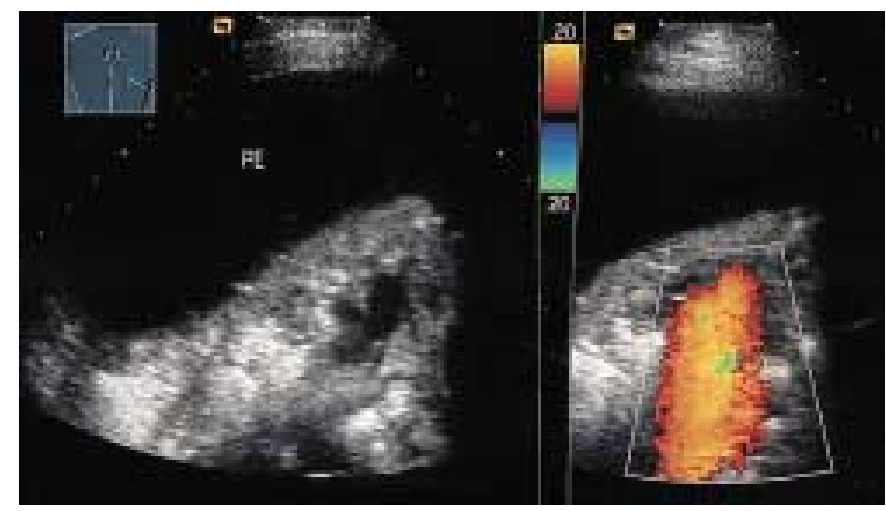

Abb. 4 85-jähriger Patient mit ausgeprägtem rechtsseitigen Pleuraerguss (PE) und Cor pulmonale. A Darstellung einer zentralen echofreien Läsion bei kompletter Unterlappenatelektase. B Farbdopplersonographisch lässt sich eine vaskuläre Raumforderung identifizieren, welche einer dilatierten Pulmonalarterie entspricht.

Fig. 485 year old patient with severe right-sided pleural effusion (PE) and cor pulmonal. A On B-mode sonography central echofree lesion was seen within atelectasis of the inferior lobe. B Colour Doppler sonography identified a vascular lesion, caused by a dilated pulmonary artery.

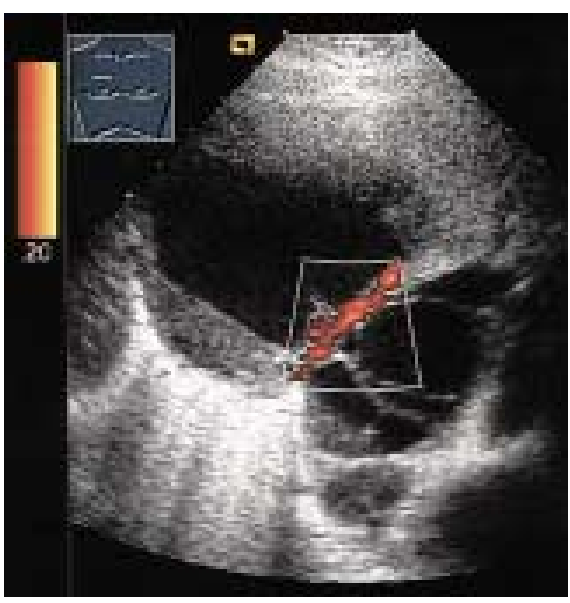

Abb. 5 44-jährige Patientin mit Liposarkom. Darstellung einer polyzystischen Raumforderung mit Gefäßstrukturen in den Septen.

Fig. 544 year old patient with liposarcoma. On power Doppler sonography an echofree septated cyst-like lesion was seen with visualisation of vasculare structures within the septs.

Differenzialdiagnostisch sind der abgekapselte Erguss im Lappenspalt, die primäre Lungenzyste, das dorsal wandständige thorakale Aortenaneurysma und seltene Gefäßanomalien (z.B. Morbus Osler) der Lunge zu erwähnen, die, falls pleurawandständig, sonographisch dargestellt werden können.

\section{Prädominant echoarme intraparenchymatöse Herde}

Hier müssen grundsätzlich echoarm-liquide von echoarm-soliden Rundherden abgegrenzt werden.

Ätiologisch liegt bei konsolidierter Lunge dem intraparenchymatös echoarm-liquiden Herd ein entzündlich einschmelzender Prozess oder ein bronchial-obstruktiver Sekretverhalt zugrunde.

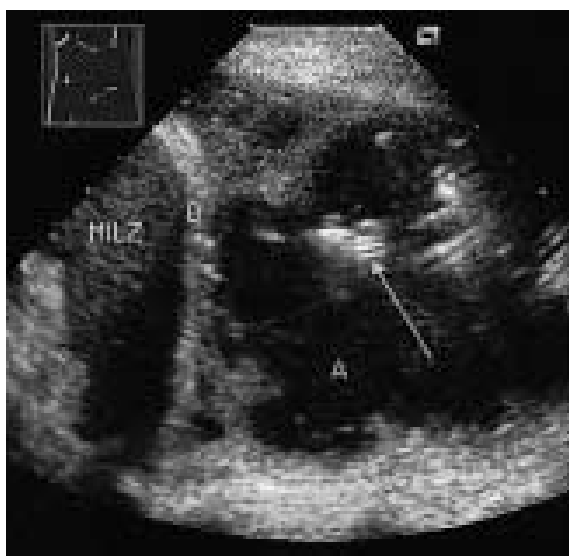

Abb. 6 77-jähriger Patient mit Alkoholkrankheit und Kachexie. Darstellung einer großen, nahezu echofreien intraparenchymatösen Raumforderung mit bewegten Luftreflexen (Pfeil) im Sinne eines Lungenabszesses (A) (über Drainagepunktion gesichert). $\mathrm{D}=$ Zwerchfell.

Fig. 677 year old patient with chronic alcoholism and cachexia. On B-mode sonography a nearby echofree liquefaction of the parenchyma was seen with air-reflexion (arrow) within the liquid area, caused by an pulmonary abscess (A). Diagnosis was confirmed by aspiration biopsy. $\mathrm{D}=$ diaphragm.

So kann es im Rahmen einer meist komplett konsolidierten Pneumonie zu vorübergehenden (Abb. 7), aber auch persistierenden Einschmelzungen kommen. Gelegentlich imponieren die Einschmelzungen multifokal mit komplexer Echogenität (Abb. 8). Bei länger bestehenden Tumorobstruktionen (obstruktive Pneumonitis) werden in der nachgeschalteten Atelektase nicht selten echoarm-liquide Raumforderungen gesehen. Hier kommen pathophysiologische pseudozystisch erweiterte bzw. destruierte Bronchiallumina mit Sekretverhalt (Abb.9) oder aber auch eine sekundäre Abszedierung infrage (Abb.10). Diagnostisch wegweisend für eine Superinfektion sind hier die Klinik sowie der eventuelle Nachweis von Luft im liquide imponierenden Areal.

Ätiologisch lässt sich beim intraparenchymatös echoarm-soliden Herd eine meist periphere Lokalisation von Lungenrundherden von einer zentralen Lokalisation von hilären Raumforderungen unterscheiden. Echoarme solide Rundherde im Sinne von Metastasen sind im Einzelfall von echoarm-liquiden Formationen auch unter Zuhilfenahme der Farbdopplersonographie (FDS) nicht sicher abzugrenzen. So zeigen auch solide Lungenrundherde im Vergleich zum umgebenden Gewebe häufig eine fehlende Gefäßdarstellung (Abb.11) oder sind wegen ausgeprägter Dyspnoe nicht mit der FDS zu charakterisieren (Abb.12). Ein echoarmer Halo, wie er sonographisch bei Lebermetastasen häufig zu finden ist, fehlt in der konsolidierten Lunge. Bei tumorbedingten Atelektasen gelingt es, durch das konsolidierte Gewebe als „akustisches Fenster“ zentrale Lungenabschnitte darzustellen und gegebenenfalls den echoarmen zentralen Tumor vom atelektatischen Lungengewebe abzugrenzen (Abb.13). 

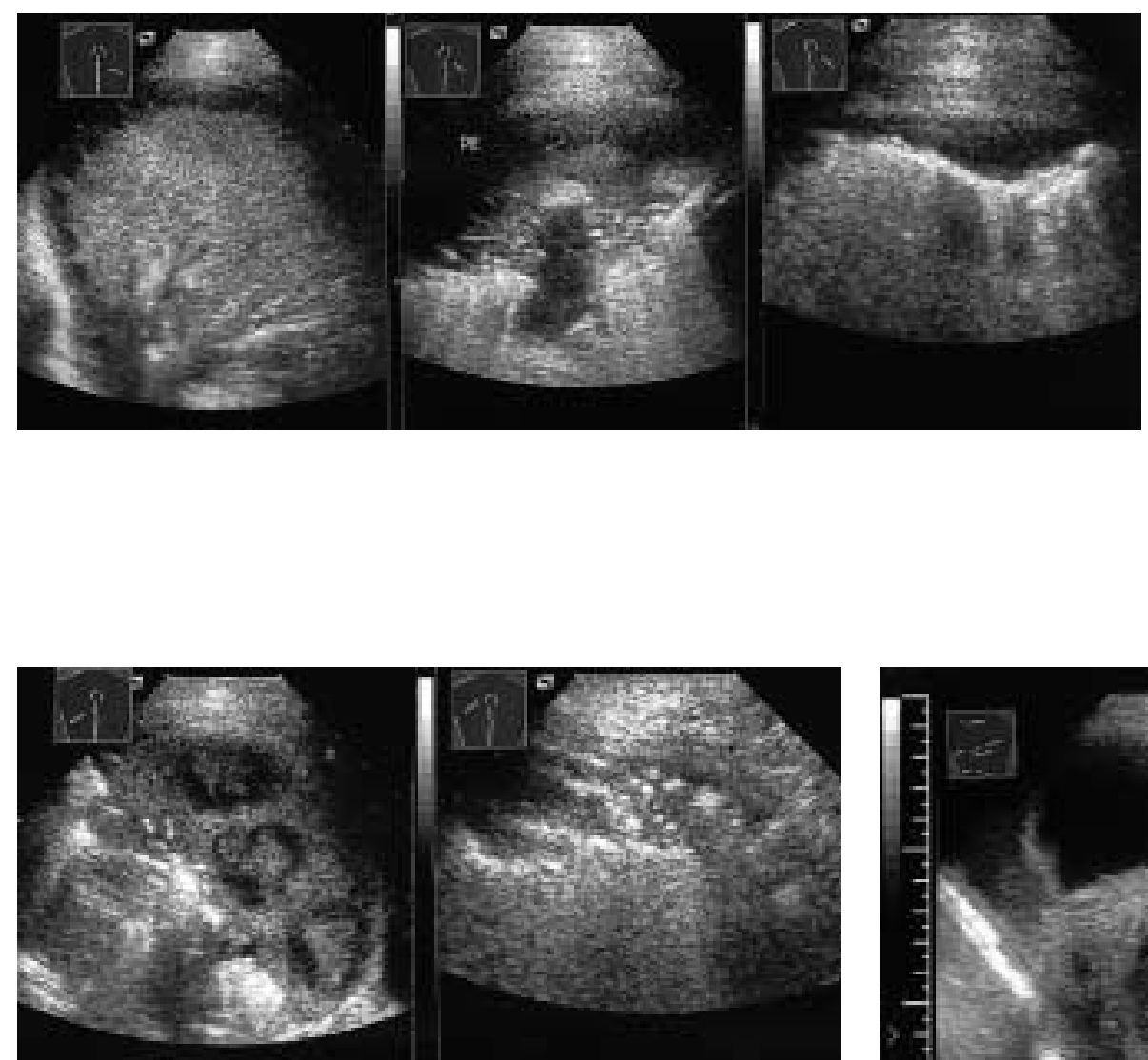

Abb. 8 23-jährige Patientin mit beatmungspflichtiger Aspirationspneumonie. A Eine Woche nach Aspiration stellen sich in der konsolidierten Lunge echogene Rundherde mit echoarmem Randsaum dar, welche am ehesten lokalisierten Abszessen entsprechen dürften. B Drei Wochen nach Aspiration ist es zu einer dramatischen Befundbesserung mit deutlicher Rebelüftung der Lunge gekommen.

Fig. 823 year old patient with aspiration pneumonia. A On B-mode sonography a nearby complete opafication of the interior lobe was seen within isoechoic round lesions and a hypoechoic "halo", caused by intra-pulmonal abscesses. B Follow-up after 3 weeks revealed a nearby complete aerated lung.

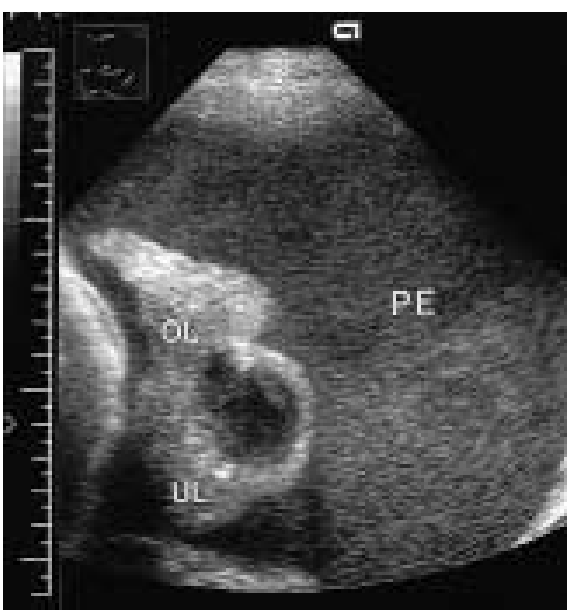

Abb. 9 64-jähriger Patient mit Bronchialkarzinom. Darstellung einer liquiden Raumforderung im Unterlappen (UL) im Sinne eines pseudozystischen Sekretverhaltes. $\mathrm{PE}=$ Pleuraerguss, $\mathrm{OL}=$ Oberlappen.

Fig. 964 year old patient with lung cancer. On B-mode sonography a hypoechoic liquid lesion was seen within the inferior lobe (UL), caused by chronic obstruction (PE= pleural effusion; $\mathrm{OL}=$ superior lobe).
Abb. 7 35-jähriger Patient mit Streptokokkenpneumonie. A Darstellung einer nahezu kompletten Hepatisation des Unterlappens. B Nach 1 Woche imponiert eine Rebelüftung mit Darstellung einer zentralen echoarmen Raumforderung (Pfeile) wie bei Einschmelzung. C Nach 2 Wochen deutliche Wiederbelüftung des Unterlappens und Rückbildung des Verhaltes.

Fig. 735 year old patient with streptococcus pneumonia. A On B-mode sonography a nearby complete "hepatisation" of the inferior lobe was seen. B Follow up after one week revealed an hypoechoic lesion (arrow) surrounded by an aerated lung caused due to liquefaction. C After 2 weeks the liquefaction resolved and a nearby complete aerated lung was seen.

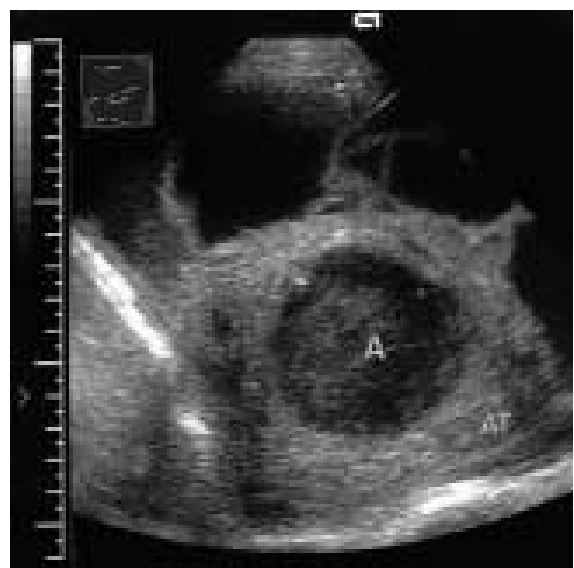

Abb. 10 53-jähriger Patient mit septischem Krankheitsbild bei Bronchialkarzinom. Zentral in der atelektatischen Lunge stellt sich ein liquider echoarmer Rundherd dar (A). Bei der Drainagepunktion konnten $120 \mathrm{ml}$ Eiter evakuiert werden.

Fig. 1053 year old patient with sepsis and lung cancer. On B-mode sonography a complete atelectasis was seen with a hypoechoic liquefaction (A). Biopsy revealed and abscess of a volume of $120 \mathrm{ml}$.

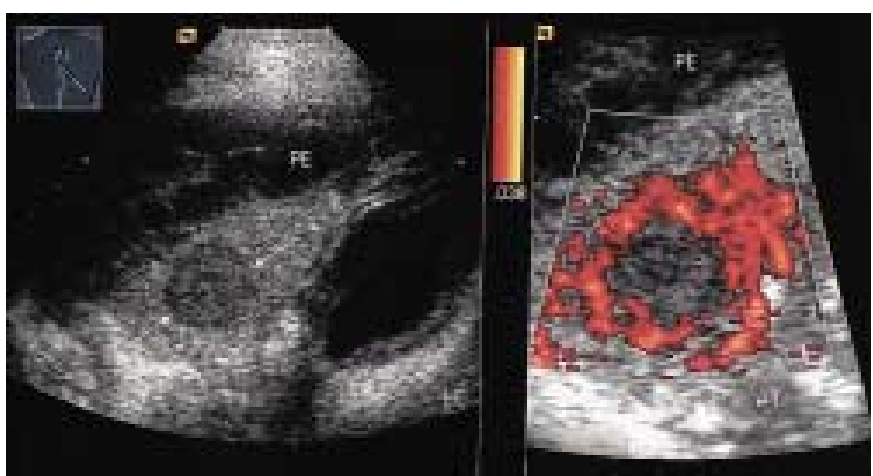

Abb. 11 73-Jähriger mit Karzinom bei unbekanntem Primärtumo mit Lungenmetastasen. A Darstellung eines echoarmen Rundherdes (Pfeile) bei Unterlappenatelektase. PE = Pleuraerguss, LE = Leber. B In der Powerdopplersonographie zeigt sich eine verstärkte Gefäßdarstellung im atelektatischen Gewebe (AT) mit Aussparung des Rundherdes. Fig. 1173 year old patient with carcinoma of unknown primary and pulmonal metastases. A On B-mode sonography an unknown hypoechoic lesion was seen with the atelectasis of the inferior lobe $(P E=$ pleural effusion; $\mathrm{LE}=$ liver). $\mathbf{B}$ On power-Doppler sonography the atelectasis (AT) had a marked vascularity. Within the lesion no flow could be detected. 


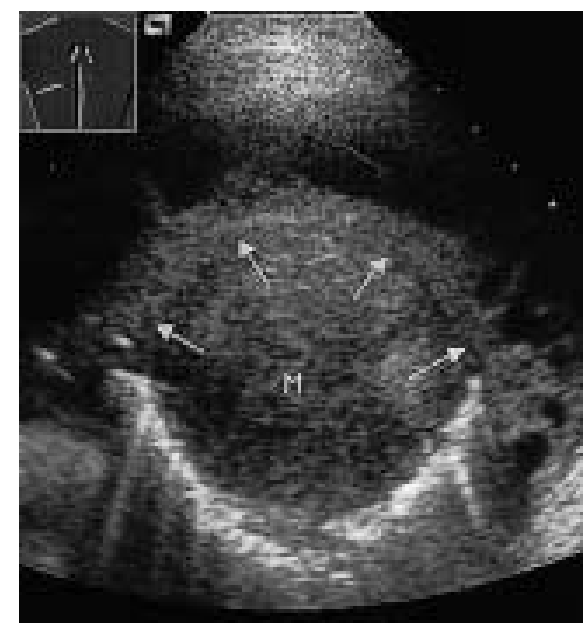

Abb. 12 62-jähriger Patient mit HNO-Karzinom und Lungenmetastasen. Darstellung eines großen echoarmen Rundherdes (M), der zu einer nachgeschalteten haubenförmigen Atelektase führt (Pfeile).

Fig. 1262 year old patient with carcinoma of the larynx and pulmonal metastases. On B-mode sonography a large hypoechoic lesion (M) was seen within the atelectasis (arrows).

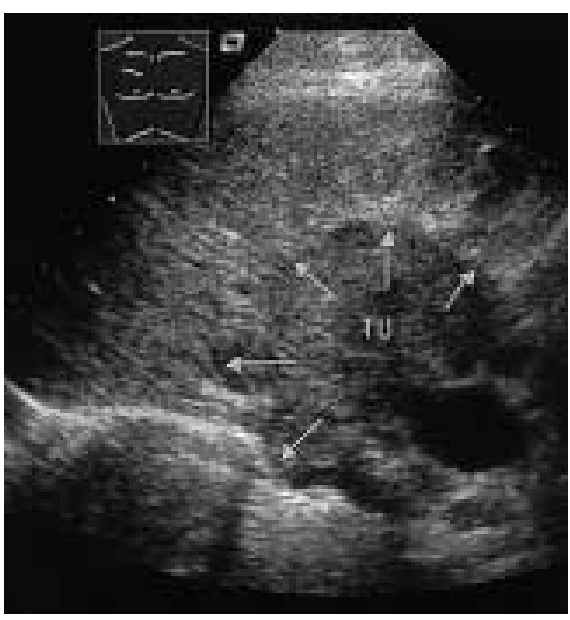

Abb. 13 70-jähriger Patient mit Bronchialkarzinom. Darstellung eines großen zentralen Tumors (TU) mit nachgeschalteter Atelektase (Pfeile).

Fig. 1370 year old patient with lung cancer. On B-mode sonography a hypoechoic central lesion (TU) with consecutive atelectasis (arrows) was seen.

\section{Prädominant echoreiche intraparenchymatöse Herde}

Pathophysiologisch liegt den fokal echoreichen Herden bei bestehender Lungenkonsolidierung in den allermeisten Fällen ein Luftverhalt in den Bronchien zugrunde. Dieser Luftverhalt, auch als „Airbronchogramm“ bezeichnet, wird typischerweise bei Pneumonien, in seltenen Fällen auch beim Bronchialkarzinom, speziell beim bronchio-alveolären Karzinom, gesehen und kennzeichnet indirekt das Ausmaß der Infiltration („Negativbild“). Es lassen sich unterschiedliche Muster von Airbronchogrammen mit vereinzelten Reflexen, astartigen Reflexen und größeren lufthaltigen Arealen sonographisch darstellen und differenzieren (Abb.14-16). Lufthaltige Einschmelzungen (Mikroabszesse) sind sonographisch von einem fokalen Airbronchogramm kaum abgrenzbar. So kann eigentlich nur der meist zentral gelegene fo-

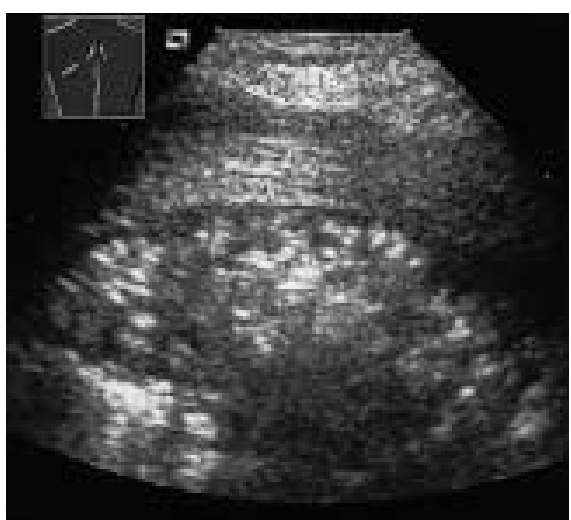

Abb. 14 51-jähriger Patient mit Pneumonie. Darstellung multipler kleinflächiger echoreicher Herdbildungen mit zeitweise dorsaler Schallauslöschung wie bei „kleinherdigem Airbronchogramm“.

Fig. 1451 year old patient with pneumonia. On B-mode sonography multiple hyperechoic foci were seen caused by sound reflexion on the aerated lung ("air bronchogram")

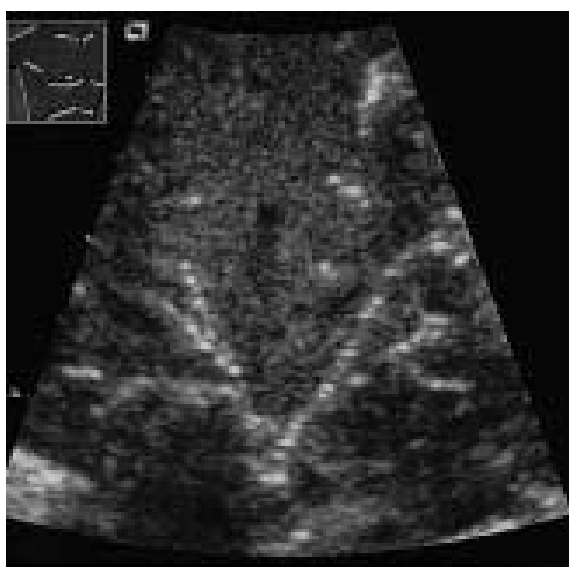

Abb. 15 73-jähriger Patient mit Pneumonie. Darstellung von länglichen echoreichen Bändern im Sinne eines „astartigen Aerobronchogramms“.

Fig. 1573 year old patient with pneumonia. On B-mode sonography multiple hyperechoic bands were seen caused by a "tree like" air bronchogram.

kal echoreiche Herd ohne atemabhängige Motilität in der komplett konsolidierten Lunge als obstruktiver und dann auch meist als entzündlicher Verhalt gewertet werden (Abb. 17) (Makroabszesse). Da hinter Luft ein „diagnostischer Totraum“ durch komplette Schallauslöschung besteht, ist zur definitiven Diagnose der luftgefüllten Abszesshöhle eine Computertomographie notwendig. Periphere pneumonische Einschmelzungsherde werden in $20-45 \%$ von Patienten mit Tuberkulose beobachtet [9]; sie sind sonographisch in der Regel gut darstellbar. Dabei kann sich das sonographische Bild der Einschmelzung im Verlauf kurzzeitig ändern, wenn der Herd Kontakt zum Bronchus bekommt (Abb. 18). Selten, in unter 1\%, können maligne Lungentumoren zentral Verkalkungen bilden, die sonographisch bei Tumorkontakt zur viszeralen Pleura dargestellt werden können [10]. 


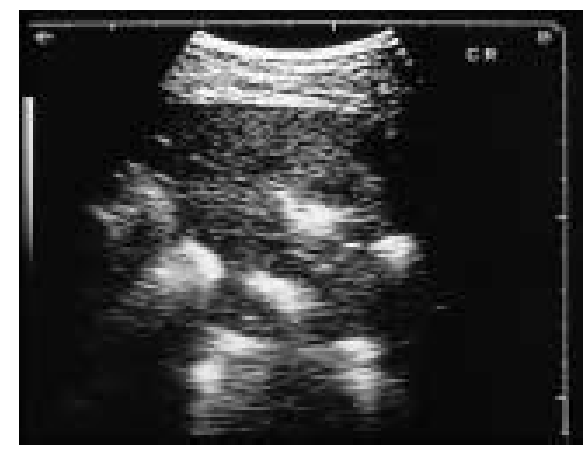

Abb. 16 30-jähriger Patient mit Sichelzellanämie und Pneumonie. Darstellung von großflächigen echoreichen Herdbildungen mit teilweiser dorsaler Schallauslöschung im Sinne eines „großherdigen Airbronchogramms".

Fig. 1630 year old patinet with sickle cell anemia and pneumonia. On B-mode sonography multiple hyperechoic foci were seen with sound reflexion caused by air broncho-gram.

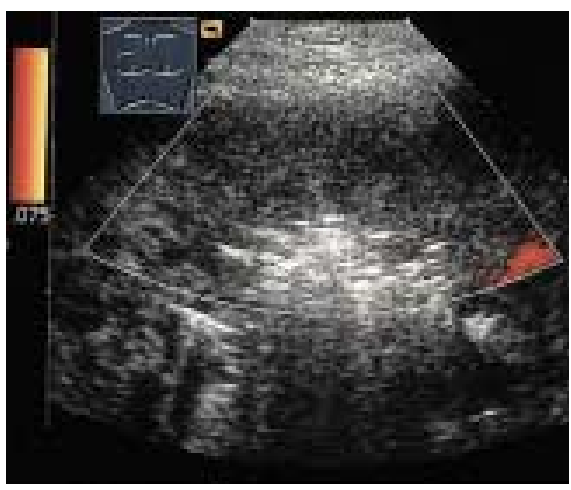

Abb. 17 52-jähriger Patient mit Bronchialkarzinom und einer bronchoskopisch gesicherten obstruktiven Oberlappenatelektase sowie Entzündungslabor. Darstellung eines zentralen echoreichen Herdes mit dorsaler Schallauslöschung. Fehlender Nachweis von Flusssignalen als Hinweis für zentralen Pulmonalarterienverschluss und Sicherung einer zentralen Einschmelzung durch Computertomographie.

Fig. 1752 year old patient with lung cancer, signs of infection and obstructive atelectasis confirmed by bronchoscopy. On power-Doppler sonography a central hyperechoic irregular demarceted lesion with sound reflexion was seen. Within the atelectasis no vascularity was seen suggesting central occlusion of the pulmonary artery. Com-puted tomopgrahy confirmed central liquefaction.

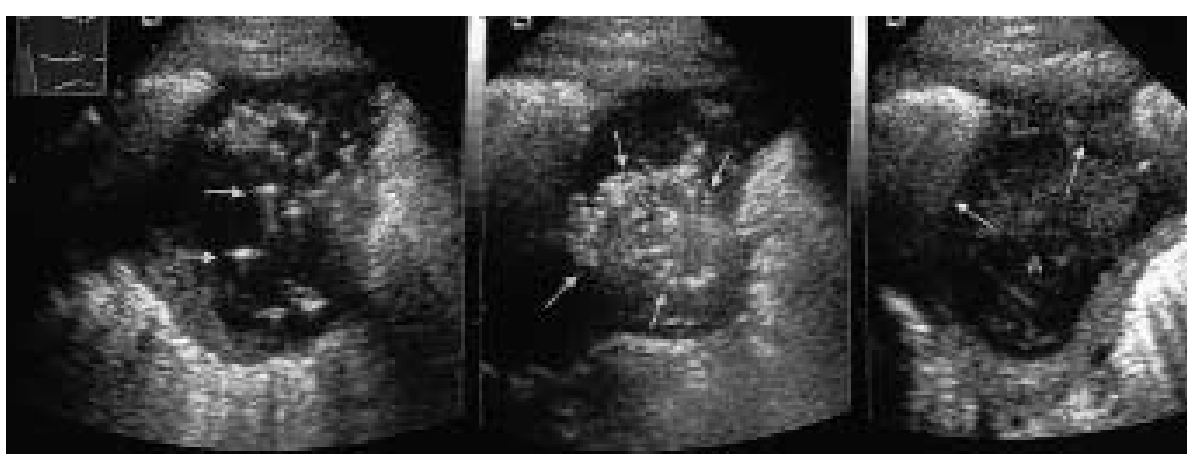

Abb. 18 40-jähriger Patient mit Alkoholkrankheit und Lungentuberkulose. A Darstellung eine Liquidefizierung mit multiplen kleinherdigen stehenden Luftreflexen (Pfeile). B Nach 3 Tagen Zunahme der echoreichen Luftreflexe (Pfeile). C Nach 7 Tagen Demarkierung einer jetzt nahezu echoarmen Abszedierung (A) mit echogener Wanddemarkierung (Pfeile).

Fig. 1840 year old patient with chronic alcoholism and tuberculosis. A On B-mode sonography a liquefaction of the lung with multiple hyperechoic gas reflexion (arrow) was seen. B Follow-up after 3 days revealed increased gas reflexion (arrows). C On followup after 7 days a nearby complete hypoechoic liquid lesion (A) with echoic borders was seen (arrows).

Zusammenfassend ist die sonographische Beurteilung von fokalen Parenchymläsionen nur im Kontext des primär zugrunde liegenden Pathomechanismus möglich. Die definitive Diagnose ist in den allermeisten Fällen durch invasive Prozeduren, wie kontrastmittelunterstützte Computertomographie, Bronchoskopie und interventionelle Maßnahmen, zu stellen.

\section{Literatur}

${ }^{1}$ Görg C, Görg K, Schwerk WB et al. Pleural effusion: An „acustic window“ for sonography of pleural metastases. JCU 1991; 19: 93 -97

2 Yang PC, Luh KT, Chang DB et al. Value of sonography in determining the nature of pleural effusion: analysis of 320 cases. AJR 1992; 159: $29-33$

${ }^{3}$ Yang PC, Luh KT, Chang DB et al. Ultrasonographic evaluation of pulmonary consolidation. Am Rev Respir Dis 1992; 146: 757-762

${ }^{4}$ Gehmacher O, Mathis G, Kopf A et al. Ultrasound imaging of pneumonia. Ultrasound Med Biol 1995; 21: 1119-1122

${ }^{5}$ Görg C, Weide R, Walters E et al. Sonographic findings in lung atelectases. Ultraschall Klin Prax 1996; 11: 14-19

${ }^{6}$ Mathis G, Dirschmid K. Pulmonary infarction: sonographic appearance with pathologic correlation. Eur J Radiol 1993; 17: 170-174

${ }^{7}$ Yuan A, Chang DB, Yu CJ et al. Color doppler sonography of benign and malignant pulmonary masses. AJR 1994; 163: 545-549

${ }^{8}$ Byrd RB, Carr DT, Miller WE et al. Radiographic abnormalities in carcinoma of the lung as relation to histological cell type. Thorax 1969; 24 : $573-579$ 